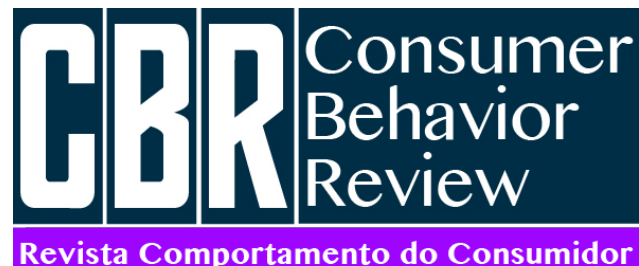

Revista Comportamento do Consumidor
Farias, M. L., Barbosa, M. L. A., Sousa Júnior, J. H., \& Silva, L. A. (2021). Valores Humanos no Consumo Colaborativo: Uma Aplicação Utilizando a Abordagem Funcionalista de Gouveia (2003). Consumer Behavior Review, 5(2), 170-181.
ISSN: 2526-7884

Editor: Prof. Dr. Marconi Freitas da Costa

E-mail: cbr@ufpe.br
Avaliação: Double blind review

Recebido: 29 de Março, 2020

Aceito: 19 de Maio, 2020

\title{
VALORES HUMANOS NO CONSUMO COLABORATIVO: UMA APLICAÇÃO UTILIZANDO A ABORDAGEM FUNCIONALISTA DE GOUVEIA (2003)
}

\author{
Human Values in Collaborative Consumption: An Application Using the Functionalist \\ Miriam Leite Farias ${ }^{1}$ \\ ORCID: http://orcid.org/0000-0002-3207-9900 \\ E-mail: miriamlfarias@gmail.com \\ Maria de Lourdes de Azevedo Barbosa ${ }^{1}$ \\ ORCID: http://orcid.org/0000-0002-1790-380X \\ E-mail: lourdesbarbosa@gmail.com \\ João Henriques de Sousa Júnior ${ }^{2}$ \\ ORCID: http://orcid.org/0000-0001-8589-8101 \\ E-mail: sousajunioreu@hotmail.com \\ Luana Alexandre Silva ${ }^{1}$ \\ ORCID: http://orcid.org/0000-0003-2288-0216 \\ E-mail: luanaalsilva@gmail.com \\ ${ }^{1}$ Universidade Federal de Pernambuco, Recife, Pernambuco, Brasil \\ ${ }^{2}$ Universidade Federal de Santa Catarina, Florianópolis, Santa Catarina, Brasil
} Approach of Gouveia (2003)

\begin{abstract}
Resumo
A temática do consumo colaborativo tem ganhado popularidade nos últimos anos não apenas na academia, mas também na sociedade. Assim, as atitudes em relação ao consumo têm mudado, mas pouco ainda se
\end{abstract}

\begin{abstract}
The theme of collaborative consumption has gained popularity in recent years not only in academia, but also in society. Thus, how attitudes towards consumption have changed, but little is discussed in the literature about
\end{abstract}


discute na literatura sobre a influência dos valores nesse tipo de consumo. Dessa forma, essa pesquisa objetivou identificar os valores humanos, a partir da teoria funcionalista de Gouveia (2003), de consumidores que se engajam em uma atividade de consumo colaborativo. Para isso, realizou-se um survey online com 208 usuários da plataforma de hospedagem Airbnb, sendo os dados analisados a partir da estatística descritiva. Os resultados apontam que as subfunções de Existência, Suprapessoal e Interação foram as mais endossadas pelos respondentes. Estes resultados apresentam contribuição inédita no entendimento dos valores humanos no contexto do consumo colaborativo e podem implicar em novas práticas para empresas como a criação de ações publicitárias mais específicas ao público deste tipo de consumo.

Palavras-chave: Consumo colaborativo; Valores humanos; Teoria Funcionalista;

Airbnb. the influence of values on this type of consumption. Thus, this research aimed to identify human values, based on the functionalist theory of Gouveia (2003), of consumers who engage in a collaborative consumption activity. For this, an online survey was carried out with 208 users of the Airbnb hosting platform, informing the data from the descriptive statistics. The results show that the sub-functions of Existence, Suprapersonal and Interaction were the most endorsed by the respondents. These results present an unprecedented contribution to the understanding of human values in the context of collaborative consumption and may imply new practices for companies such as the creation of public actions more specific to the public of this type of consumption.

Keywords: Collaborative consumption; Humans values; Functionalist theory; Airbnb.

This work is licensed under a Creative Commons Attribution 4.0 International License.

\section{INTRODUÇÃO}

0 consumo colaborativo tem ganhando popularidade nos últimos anos devido às recentes crises econômicas mundiais (Tussyadiah, 2015) e, apesar de ser um fenômeno recente, já é considerada uma forma de negócio bastante popular, principalmente devido a facilidade do acesso à web e suas plataformas (Sousa Júnior, 2018). Por meio desse fenômeno, a posse de um produto é substituída por seu acesso temporário (Bostman \& Rogers, 2010). Logo, seus impactos estão reinventando a organização do mercado tradicional (Santoso \& Erdaka, 2015).

Belk (2014) o define como uma atividade em que pessoas coordenam a aquisição e a distribuição de recursos em troca de uma taxa ou outra compensação. No consumo colaborativo as duas partes procuram igualmente se ajudar, como uma maneira de reduzir custos, tempo e esforço, tornando a troca entre os indivíduos mais fácil e rapidamente acessível (Bostamn \& Rogers, 2010).

Para Bostman e Rogers (2010), o compartilhamento e a colaboração estão acontecendo em uma escala nunca antes possível e o consumo colaborativo é uma ideia "bola de neve" que continuará crescendo e ganhando força nos próximos anos, ultrapassando uma simples tendência. Isso porque o movimento permite que as pessoas percebam os benefícios do acesso temporário aos produtos no lugar de ter a posse, e, na medida que fazem isso, economizam dinheiro, espaço e tempo, fazem novos amigos e se tornam cidadãos mais ativos. E em se tratando dos bens, tanto os produtos pertecentes a uma empresa podem ser compartilhados ou alugados, quanto os de propriedade privada.

Um fator importante que influenciou o desenvolvimento desse novo tipo de consumo em todo o mundo foi o avanço da Internet e das técnicas da web 2.0 (Belk, 2007, 2014). De acordo com Belk (2007), a Internet nos leva a uma etapa de compartilhamento que fornece um acesso igual a um fluxo de informações que é gratuito. Plataformas on-line tornaram possível compartilhar uma escala mais ampla (Cohen \& Kietzmank, 2014), mais conveniente e transparente, onde milhões de pessoas têm acesso em todo o mundo (Kathan, Matzaler \& Veider, 2016).

Tendo em vista que as atitudes em relação ao consumo têm mudado nos últimos anos devido à uma crescente preocupação com os impactos ambientais e sociais no planeta (Hamari, Sjökilnt \& Ukkonen, 2016), compreende-se que é necessário analisar as razões por trás do envolvimento dos 
consumidores em atividades de compartilhamento e colaboração, abrangendo a complexidade dos determinantes que influenciam no seu comportamento. Estudos recentes têm focado na identificação dos determinantes que levam os consumidores a se engajarem em práticas de consumo colaborativo (Hamari, O Sjöklint \& Ukkonen, 2016; Möhlmann, 2015; Piscicelli, Cooper \& Fisher, 2015; Tussyadiah, 2015; Silva \& Barbosa, 2018).

No entanto, ainda pouco se sabe sobre os processos subjacentes para determinar a influência dos valores nesse tipo de consumo (Ross \& Hahn, 2017). Três pesquisas recentes que relacionaram os valores humanos dos consumidores e o construto de consumo colaborativo. Inicialmente, Piscicelli, Cooper e Fisher (2015) e Martin e Upham (2015) se embasaram na teoria de Valores de Schwartz (1992, 1994, 2006) utilizando o Questionário de Valor de Retrato (PQV - Portrait Value Questionnaire) de Schwartz (2006). Já o estudo de Ross e Hahn (2017) examinou os efeitos que um padrão de consumo compartilhado tem nos valores, atitudes e normas dos consumidores, utilizando como base a teoria do comportamento planejado e a teoria do valor-norma.

A maioria dos pesquisadores refere-se ao modelo de Schwartz em diversos contextos de estudos sobre valores, utilizando, por exemplo, os instrumentos de mensuração de Schwartz Value Survey (SVS) e o questionário de valor de retrato (PVQ). Embora a teoria universal dos valores humanos com os dez tipos motivacionais propostos por Schwartz seja a mais comumente aceita, ainda apresenta suas limitações.

Gouveia (1998, 2003), por sua vez, com base nos modelos existentes, propôs uma teoria denominada de Teoria Funcionalista dos Valores Humanos. Ao comparar os dois modelos percebe-se que o de Gouveia $(1998,2003)$ apresenta-se de forma mais completa, obtendo melhores resultados tanto na adequação da hipótese de estrutura quanto no poder explicativo, demonstrando maior correlação média com os indicadores comportamentais.

Considerando isto, o objetivo deste estudo foi identificar os valores humanos (Gouveia, 2003) de consumidores que se engajam em uma atividade de consumo colaborativo. Para tanto, foi aplicado um survey com usuários da plataforma de aluguel de hospedagens Airbnb.

\section{VALORES HUMANOS RELACIONADOS AO CONSUMO COLABORATIVO}

Embasados na teoria de Valores de Schwartz $(1992,1994,2006)$ e utilizando o Questionário de Valor de Retrato (PQV - Portrait Value Questionnaire) de Schwartz (2006), Piscicelli, Cooper e Fisher (2015) verificaram que benevolência e universalismo eram prioridades para os respondentes de sua pesquisa, e que os valores de autotranscedência e abertura à mudança pontuaram melhor em contraste com os valores de autopromoção e conservação. Os autores sugerem que a Tradição (preservar bens culturais ou familiares e tradições religiosas) é um valor que pode ameaçar o desenvolvimento do Consumo Colaborativo na medida que os modelos alternativos alteram o status $q u o$, trazendo inovações que desafiam os modelos tradicionais (Piscicelli et al., 2015).

Martin e Upham (2015) também destacaram os valores de auto transcendência e abertura à mudança, no entanto, o valor Tradição foi bem pontuado pelos participantes, ao contrário do que aconteceu no estudo de Piscicelli et al. (2015). Os autores mesclaram a teoria de Schwarz et al. (2012) com a Teoria da incorporação coletiva de valores de Chen, Lune e Queen (2013), e ao fim, desenvolveram um modelo conceitual que testaram com usuários de grupos de re-uso. Os grupos de re-uso também são considerados uma forma de Consumo Colaborativo (Bostaman \& Rogers, 2010), pois permitem que as pessoas doem diretamente algo que não utilzam mais, à alguém que precisa daquilo e está localizado na mesma área.

Diferentemente da compreensão obeservada na literatura, de que os valores poderiam influenciar o comportamento, Ross e Hahn (2017) partiram da ideia contrária, procurando responder que efeitos o consumo compartilhado (shared consumption) tem nos valores, atitudes e normas dos consumidores, implicando em uma influência causal do comportamento nesses fatores sóciopsicológicos. Os autores se basearam na teoria do comportamento planejado de Ajzen $(1985,1991)$ e na teoria da norma-valor-crença de Stern et al. (1999) para aplicar um estudo cross-sectional de nove meses, onde as mudanças nas variáveis, influências causais e efeitos habituais seriam mensurados. 
No final, foi achado que as relações entre valores, atitudes e normas mutualmente causam efeitos umas nas outras em um processo recíproco e contínuo, sugerindo que quanto mais os consumidores estão envolvidos no comportamento de consumo compartilhado, mais altruístas se tornam com o tempo. Além disso, o maior envolvimento no consumo compartilhado leva os consumidores a manterem atitudes, normas subjetivas e normas pessoais mais favoráveis a esse tipo de consumo.

Arruda et al. (2016), por sua vez, analisaram as relações entre os valores pessoais dos consumidores e o consumo colaborativo de bicicletas compartilhadas na cidade Fortaleza - CE. Os autores destacaram como motivações para a realização do consumo colaborativo de bicicletas, a economia de custos e tempo, a consciência socioambiental e crença no bem comum. Identificaram os valores pessoais de Autodeterminação e Estimulação, referentes à dimensão abertura à mudança, como sendo os mais destacados, assim como, o perfil dos consumidores realizando uma análise de clusters: usuários jovens e solteiros, com renda intermediária. Por fim, acharam que os valores pessoais dos consumidores de bicicleta compartilhada influenciam o consumo colaborativo.

Em suma, após essa revisão de estudos anteriores envolvendo os valores humanos dos consumidores engajados em atividades colaborativas, percebe-se que esse construto, ainda que ínfimamente, ja fora trabalhado na literatura, no entanto, não foram encontradas referências da Teoria Funcionalista proposta por Gouveia $(1998,2003)$, indicando a contribuição teórica que este estudo propõe-se a dar a partir de seus resultados.

Com relações aos achados dos trabalhos encontrados, destacam-se a presença de motivações relacionadas à poupança de custos, sustentabilidade e interação com a comunidade. Os valores humanos mais endossados por esses consumidores fazem parte da dimensão de abertura à mudanças e auto-transcedência, quando utilizada a abordagem teórica dos tipos motivacionais de Schwartz (1992, 1994). Em maioria, os autores utilizaram o Questionário de Valor de Retrato (PQV - Portrait Value Questionnaire) de Schwartz (2006).

\section{TEORIA FUNCIONALISTA DOS VALORES HUMANOS}

Este estudo utilizou uma tipologia mais recente sobre valores, conhecida como Teoria Funcionalista dos Valores Humanos. Gouveia (2003, p. 433) define os valores como "categorias de orientação que são desejáveis, com base nas necessidades humanas e precondições para satisfazê-las, que são adotadas pelos atores sociais, e que podem variar em sua magnitude e seus elementos constituintes" e as funções dos valores são características psicológicas de comportamentos oriendados e representações das necessidades humanas (Gouveia, 2013). 0 modelo criado pelo autor assume quatro pressupostos: (1) o homem tem a natureza benevolente (Maslow, 1954), onde os valores são positivos e desejáveis (Kluckhon, 1951); (2) os valores são princípios guias da vida do indivíduo (Rockeach, 1973); (3) os valores tem base motivacional (Inglehart,1977; Maslow, 1954) e (4) são tratados como terminais, representando metas individuais superiores (Rockeach, 1973) (Gouveia et al., 2011; Athayde, 2012 ).

De acordo com a Teoria Funcionalista, as funções formam duas dimensões diferentes. A primeira dimensão descreve o "círculo de metas" baseado na orientação dos valores que guiam o comportamento humano (pessoais, centrais ou sociais) e a segunda dimensão define o "nível de necessidades" com base no tipo de motivador de valores que representam cognitivamente as necessidades humanas (materialista ou abstrata) (Gouveia et al., 2014; Gouveia et al., 2015).

A primeira dimensão diferencia os valores de acordo com sua orientação, sendo ela pessoal, social ou central (Gouveia et al., 2014; Gouveia et al., 2015). Os valores pessoais são subdividos em de experimentação e realização, os sociais, em normativos e de interação, e finalmente, os centrais, em valores de existência e suprapessoais (Gouveia, 1998, 2003).

A segunda dimensão diz respeito a expressão das necessidades dos indivíduos. Na literatura, os valores podem ser classificados com necessidades materialistas (pragmáticos) ou idealistas (abstratos) (Iglehart, 1977; Maslow, 1954; Welzel, Inglehart \& Kligemann, 2003). Os valores materialistas/pragmáticos expressam necessidades biológicas e sociais, tais como se alimentar e questões de segurança, eles visam, por exemplo, garantir a sobrevivência do indivíduo. Condizem com 
uma orientação para seguir metas e regras normativas. Enquanto os valores idealistas/abstratos expressam uma orientação baseada em princípios mais abstratos, quando já satisfeitas as necessidades básicas. Os indivíduos que possuem esses valores possuem uma maior abertura à mudanças e criatividade, não tendo tanta dependência de bens materiais ou objetos concretos (Maslow, 1954; Iglehart, 1977; Braithwaite et al., 1996).

Logo, a segunda dimensão dos valores os diferencia de acordo com as necessidades humanas, sendo o tipo motivador materialista ou idealista. (Gouveia et al., 2014; Gouveia et al., 2015). As duas funções valorativas descritas formam dois eixos principais que representam a estrutura espacial dos valores. 0 eixo horizontal condiz com as metas que orientam o comportamento (pessoal, social ou central) e o eixo vertical com o tipo motivador, as necessidades que os valores expressam (materialistas ou idealistas). A interação, ou seja, o cruzamento entre os dois eixos gera um framework 3x2 com seis subfunções e valores básicos específicos (Gouveia et al., 2015; Gouveia, 1998, 2003). A Figura 1 apresenta uma descrição esquemática dessa interação.

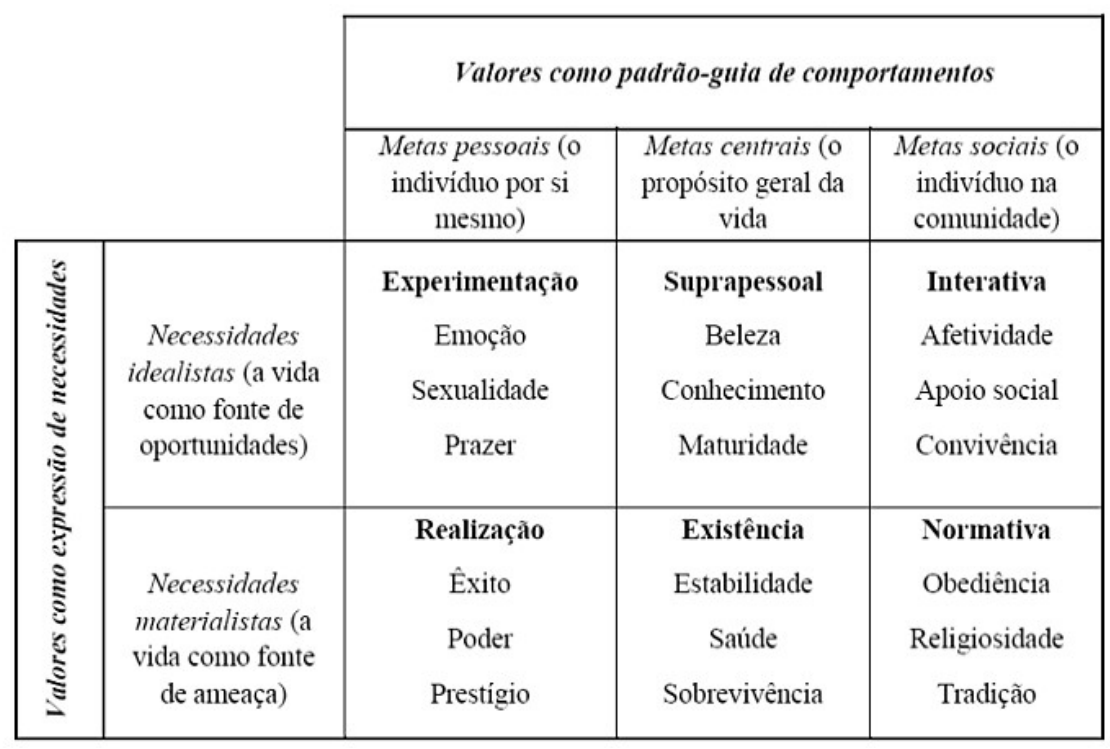

Fonte: Adaptado de Gouveia et al. (2014)

Figura 1. Facetas, dimensões e valores básicos

Conforme mostra a Figura 1, a combinação das duas principais funções, gera seis subfunções diferentes. 0 eixo horizontal apresenta cada um dos três tipos de metas perseguidas que são representadas por três subfunções: pessoal (valores de experimentação e realização), central (valores suprapessoal e existência) e social (valores de interação e normativo). Já no eixo vertical, cada um dos dois tipos de necessidades expressas é representado por duas subfunções: materialista (valores de realização, existência e normativo) e abstrata (valores de experimentação, suprapessoais e interação) (Gouveia et al., 2015). As especificidades de cada uma das sub-funções são apresentadas a seguir.

\section{Subfunções dos valores e suas características}

(a) A subfunção de existência (central/materialista) postula que os valores são compatíveis com objetivos pessoais e sociais dentro da expressão de necessidades de sobrevivência, pois seu propósito é assegurar as condições básicas de sobrevivência biológica e psicológica individual. A teoria postula que os valores de existência são referência para valores de realização e valores normativos (Gouveia et al., 2014; Gouveia et al., 2015).

(b) A subfunção de realização (pessoal/materialista) expressa as necessidades de sobrevivência e cumprimento metas. Tais valores também são importantes para interações sociais bemsucedidas e funcionamento institucional (Schwartz, 1992). Indivíduos orientados pelos valores de realização dão importância à hierarquia e a enxergam como demonstração de competência 
pessoal. Valorizam também uma sociedade estruturada e seus próprios benefícios pessoais, são geralmente considerados decisores práticos (Gouveia et al., 2014; Gouveia et al., 2015).

(c) A subfunção Normativa (social/materialista) expressa necessidades de sobrevivência, mas com foco em metas sociais. Os valores normativos representam necessidade de Segurança e Controle, bem como as condições prévias para a necessidades básicas. Esses valores têm uma orientação social com foco nas regras sociais, baseados em principios materialistas. Os individuos orientados por esses valores dão importância à manutenção da cultura e das normas. Enfatizam também a obediência à autoridade (Gouveia et al., 2014; Gouveia et al., 2015).

(d) A subfunção Suprapessoal (central/idealista) assim como os valores de existência, foca em metas centrais e necessidades idealistas. Representam necessidades como estética, de cognição e de auto-realização. Estabelecem clareza e estabilidade na categorização do mundo de uma forma cognitiva (Gouveia et al., 2011). São um ponto de referência para valores de excitação e valores interativos (Gouveia et al., 2014; Gouveia et al., 2015).

(e) A subfunção de Experimentação (pessoal/ idealista) possui valores com necessidade de satisfação e prazer (hedonismo), que contribuem para mudanças e inovações no status quo (Gouveia, 2013). Os indivíduos que possuem esses valores geralmente são jovens, que não buscam metas fixas ou materiais a longo prazo (Gouveia et al., 2014; Gouveia et al., 2015).

(f) A subfunção Interativa (social/ idealista) compreende necessidades de manutenção das relações interpessoais. 0 contato social é um objetivo dos indivíduos guiados por valores interativos. Representam necessidade de pertencimaneto, de amor e afiliação . As pessoas guiadas por estes valores são mais jovens e orientadas para relações estáveis (Milfont, Gouveia \& Costa, 2006) (Gouveia et al., 2014; Gouveia et al., 2015).

Em suma, a Teoria Funciolista dos Valores Humanos tem a intenção de integrar modelos anteriores, apresentando uma solução parcimoniosa sobre os estudos dos valores humanos (Gouveia et al., 2015). Ela é construída com base na fundamentação teórica das ideias iniciais da teoria da ação de Parsons (1959) e Kluckhohn (1951), considerando também a abordagem psicológica de Maslow (1954), e finalmente, acrescentando os modelos mais recentes de Inglehart (1977) e Schwartz (1992, 1994).

\section{PROCEDIMENTOS METODOLÓGICOS}

Para os fins desta pesquisa foi realizado um survey online com 208 consumidores que já utilizaram ao menos uma vez os serviços do Airbnb, uma plataforma de hospedagens do consumo colaborativo. A operacionalização de coleta dos dados se deu por meio da aplicação de questionários durante os meses de outubro a dezembro de 2017.

O questionário foi criado utilizando a ferramenta gratuita do Google Forms e seu link foi disponibilizado em nas redes sociais virtuais, especificamente em páginas relativas a discussões sobre plataformas de consumo colaborativo, como a página do Facebook "Airbnb Brasil". Desse modo, a amostra foi composta de modo não intencional e não probabilística. É imprescindível salientar que o instrumento de coleta passou por validação de três acadêmicos da área, todos com titulação mínima de doutor e pesquisadores em comportamento de consumo e consumo colaborativo, e foi também submetido à pré-teste durante o mês de setembro de 2017, o que possibilitou feedbacks para melhoria da compreensão dos itens, só então resultando na versão final do questionário.

0 questionário proposto por Gouveia e seus colaboradores vem sendo aperfeiçoado ao longo dos anos. Foi inicialmente desenvolvido com 66 itens (Gouveia, 1998), seguido de uma versão abreviada com 24 itens (Gouveia, 2003) e finalmente reduzido para sua versão final com 18 itens (Gouveia et al., 2008), o qual foi utilizado para esta pesquisa. Os 18 itens analisados na pesquisa estão dispostos na Tabela 1. 
Tabela 1

Itens do Questionário de Valores Humanos

\begin{tabular}{|c|c|}
\hline Item & Objetivo \\
\hline VH1: Sexualidade. & Ter relações sexuais; obter prazer sexual. \\
\hline VH2: Êxito. & Obter o que se propõe; ser eficiente em tudo que faz. \\
\hline VH3: Apoio social. & $\begin{array}{l}\text { Obter ajuda quando a necessita; Sentir que não está } \\
\text { só no mundo. }\end{array}$ \\
\hline VH4: Conhecimento. & $\begin{array}{l}\text { Procurar notícias atualizadas sobre assuntos pouco conhecidos; } \\
\text { tentar descobrir coisas novas sobre o mundo. }\end{array}$ \\
\hline VH5: Emoção. & Desfrutar desafiando o perigo; buscar aventuras. \\
\hline VH6: Poder. & $\begin{array}{l}\text { Ter poder para influenciar os outros e controlar decisões; ser } \\
\text { um chefe de uma equipe. }\end{array}$ \\
\hline VH7: Afetividade. & $\begin{array}{l}\text { Ter uma relação de afeto profunda e duradoura; ter alguém para } \\
\text { compartilhar seus êxitos e seus fracassos. }\end{array}$ \\
\hline VH8: Religiosidade. & $\begin{array}{l}\text { Crer em Deus como salvador da humanidade; cumprir a vontade } \\
\text { de Deus. }\end{array}$ \\
\hline VH9: Saúde. & $\begin{array}{l}\text { Preocupar-se com a saúde antes mesmo de ficar doente; não } \\
\text { estar enfermo. }\end{array}$ \\
\hline VH.10: Prazer. & Desfrutar da vida; satisfazer todos os seus desejos. \\
\hline VH11: Prestígio. & $\begin{array}{l}\text { Saber que muita gente lhe conhece e lhe admira; quando mais } \\
\text { velho receber uma homenagem por suas contribuições. }\end{array}$ \\
\hline VH12: Obediência. & $\begin{array}{l}\text { Cumprir seus deveres e obrigações do dia a dia; respeitar seus } \\
\text { pais, os superiores, e os mais velhos. }\end{array}$ \\
\hline VH13: Estabilidade social. & $\begin{array}{l}\text { Ter certeza que amanhã terá tudo que tem hoje; ter uma vida } \\
\text { organizada e planificada. }\end{array}$ \\
\hline VH14: Convivência. & $\begin{array}{l}\text { Conviver diariamente com os vizinhos, fazer parte de algum } \\
\text { grupo como: grupo social, religioso, esportivo, entre outros. }\end{array}$ \\
\hline VH15: Beleza. & $\begin{array}{l}\text { Ser capaz de apreciar o melhor da arte, música e literatura, ir a } \\
\text { museus ou exposições onde possa ver coisas belas. }\end{array}$ \\
\hline VH16: Tradição. & $\begin{array}{l}\text { Seguir as normas sociais do seu país; respeitar as tradições da } \\
\text { sua sociedade. }\end{array}$ \\
\hline VH17: Sobrevivência. & $\begin{array}{l}\text { Ter água, comida e poder dormir bem todos os dias; viver em } \\
\text { um lugar com abundância de alimentos. }\end{array}$ \\
\hline VH18: Maturidade. & $\begin{array}{l}\text { Sentir que conseguiu alcançar seus objetivos na vida; } \\
\text { desenvolver todas as suas capacidades. }\end{array}$ \\
\hline
\end{tabular}

Fonte: Adaptado de Gouveia et al. (2008).

Trata-se de um instrumento contendo os dezoito valores básicos com dois descritivos por valor. Os participantes avaliam cada item considerando a importância de cada valor como princípioguia para suas vidas. Os valores são medidos em uma escala tipo Likert que varia de 1 (Totalmente não importante) a 7 (Extremamente importante). As seis subfunções valorativas (Experimentação, Realização, Interação, Normativa, Suprapessoal e Existência), apresentam índices de confiabilidade aceitáveis, variando de 0,53 a 0,60 no coeficiente de alfa de Cronbach (Gouveia et al., 2014).

\section{ANÁLISE DOS DADOS}

Como mostra a Tabela 2, os participantes da pesquisa são jovens adultos, em sua maioria moradores da região Nordeste, solteiros, com nível de escolaridade e renda mensal familiar considerados altos. As duas últimas características, escolaridade e renda, são semelhantes nos perfis achados de outros estudos com o tema consumo colaborativo (Möhlmann, 2015; Böcker \& Meelen, 2016; Arruda et al., 2016). A variável de idade modifica bastante entre os estudos encontrados durante a revisão, não sendo apenas jovens os usuários destes serviços. 
Tabela 2

Perfil sociodemográfico dos respondentes

\begin{tabular}{l|l|l}
\hline \multicolumn{2}{l|}{ Características demográficas } & Amostra \\
\hline \multirow{2}{*}{ Sexo } & $\begin{array}{l}\text { Feminino } \\
\text { Masculino }\end{array}$ & $\begin{array}{l}54,80 \% \\
45,20 \%\end{array}$ \\
\hline \multirow{2}{*}{ Idade } & $\begin{array}{l}\text { Média } \\
\text { DP }\end{array}$ & $\begin{array}{l}29,9 \\
9,1\end{array}$ \\
\hline Local de residência & Nordeste & $60,60 \%$ \\
\hline Estado civil & Solteiro & $70,20 \%$ \\
\hline Escolaridade & Superior completo & $39,90 \%$ \\
\hline \multirow{2}{*}{ Renda familiar mensal (R\$) } & Média & $8.653,29$ \\
& DP & $7.823,45$ \\
\hline \multirow{2}{*}{ Frequência de uso } & Utilizou apenas uma vez & $47,10 \%$ \\
& Utilizou mais de uma vez & $52,90 \%$
\end{tabular}

Fonte: Dados da pesquisa.

Também foi realizado o cálculo do coeficiente de alfa de Cronbach (Malhotra, 2006) das subfunções valorativas. Tais resultados são apresentados na Tabela 3. De acordo com os padrões de referência para o teste, o valor mínimo aceitável para indicar uma confiabilidade satisfatória é 0,60, abaixo desse valor a consistência interna da escala utilizada é considerada baixa (Cronbach, 1951).

Tabela 3

Índices de consistência interna das escalas

\begin{tabular}{l|c|c}
\hline Escalas & $\begin{array}{c}\text { Quantidade de } \\
\text { itens }\end{array}$ & alfa de Cronbach \\
\hline Subfunção Experimentação & 3 & 0,473 \\
Subfunção Suprapessoal & 3 & 0,404 \\
Subfunção Interação & 3 & 0,582 \\
Subfunção Realização & 3 & 0,671 \\
Subfunção Existência & 3 & 0,57 \\
Subfunção Normativa & 3 & 0,678 \\
\hline
\end{tabular}

Fonte: Dados da pesquisa. Nota. $\alpha=$ alfa de Cronbach.

Como podemos observar na Tabela 3, os valores de alfa de Cronbach considerando as seis subfunções valorativas, variam de 0,404 a 0,678 , sendo valores de $\alpha$ relativamente aceitáveis. 0 objetivo deste estudo foi identificar os valores humanos mais pontuados pelos consumidores que utilizam os serviços do Airbnb. Para isso, foram realizadas estatísticas descritivas (média e desvio padrão) das seis subfunções no intuito de avaliar os índices decorrentes dos escores das prioridades valorativas.

Conforme apresentado na Tabela 4, as subfunções mais endossadas pelos respondentes foram a de Existência, Suprapessoal e Interação, seguidas por Experimentação, Realização e Normativa. A subfunção de Existência corresponde às representações cognitivas das necessidades fisiológicas básicas, como comer e dormir. Ela é representada pelos valores de estabilidade social, saúde e sobrevivência, e é compatível com orientações sociais e pessoais dentro do domínio motivador materialista. 
Tabela 4

Média das subfunções dos valores humanos dos participantes

\begin{tabular}{l|l|l|l}
\hline \multicolumn{2}{l|}{} & Média & Desvio Padrão \\
\hline \multirow{4}{*}{$\begin{array}{l}\text { Valores } \\
\text { Humanos }\end{array}$} & Experimentação & 5,29 & 0,99 \\
\cline { 2 - 4 } & Interação & 5,54 & 0,97 \\
\cline { 2 - 4 } & Suprapesoal & 5,75 & 0,79 \\
& Realização & 5,01 & 1,07 \\
& Normativa & 4,39 & 1,43 \\
& Existência & 5,87 & 0,88 \\
\hline
\end{tabular}

Fonte: Dados da pesquisa

Os valores da subfunção de Existência são endossados principalmente por pessoas que consideram a economia do valor a ser pago no Airbnb como de valia quando analisada a questão do custo e do benefício, tendo em vista que essa subfunção é considerada a que possui maior relevância para o tipo motivador materialista. Isso demonstra que, apesar da alta média na renda mensal dos respondentes, há uma preocupação por poupar e economizar recursos em situações supérfluas, isto é, não essenciais, como em uma viagem. Esse achado fortalece o argumento encontrado na literatura que o consumo colaborativo teve sua origem a partir das consequências das recentes crises econômicas mundiais, sendo fortemente motivado por razões mais racionais, como a poupança de custos (Böcker \& Meelen, 2017; Möhlmann, 2015; Tussyadiah, 2015).

A subfunção Suprapessoal é composta pelos valores: beleza, conhecimento e maturidade. Estes valores contribuem para a visão do mundo de uma forma consistente, assim como também para a organização da estrutura cognitiva dos indivíduos. Essa subfunção representa a necessidade de autorrealização, estética e cognição. Pessoas que priorizam os valores dessa subfunção possuem uma visão de mundo mais ampla.

Já a subfunção Normativa que obteve a menor média de escores, é representada pelos valores obediência, religiosidade e tradição. Esses valores também possuem um motivador materialista, mas uma orientação social, pois refletem a importância de preservação de normas sociais e tradições culturais, e obediência às autoridades. Os valores da subfunção normativa são geralmente endossados por pessoas mais idosas que visam cumprir normas e apresentam condutas mais convencionais.

Esse achado pode ser relacionado ao que sugerem Piscicelli et al. (2015), que a Tradição (preservar bens culturais ou familiares e tradições religiosas) é um valor que pode ameaçar o desenvolvimento do Consumo Colaborativo na medida que os modelos alternativos alteram o status $q u o$, trazendo inovações que desafiam os modelos tradicionais.

\section{CONCLUSÕES}

O objetivo deste artigo foi utilizar a perspectiva da teoria funcionalista de Gouveia (2003) para identificar os valores humanos de consumidores que faziam uso dos serviços do Airbnb, uma plataforma de aluguel de acomodações pertinente ao contexto do consumo colaborativo. Estima-se que o objetivo proposto tenha sido alcançado, oferecendo contribuições teóricas e práticas.

No tocante às contribuições acadêmicas, ressalta-se o ineditismo de trazer a discussão dos valores humanos para o contexto do comportamento de consumo colaborativo, o que pode significar um novo caminho para diversas outras pesquisas nesta temática; enquanto contribuições gerenciais, os resultados aqui apresentados podem ser utilizados por gestores de plataformas de consumo colaborativo ou mesmo por participantes que estejam dispostos a compartilharem bens (imóveis, automóveis ou outros produtos) para que direcionem de maneira mais eficaz e eficiente suas ações de promoção, possibilitando a captação de mais pessoas.

As subfunções mais endossadas pelos respondentes desta pesquisa, considerando a teoria funcionalista, foram a de Existência, Suprapessoal e Interação, seguidas por Experimentação, Realização e Normativa. Esse achado corrobora com os argumentos que a poupança de custos é uma forte motivação para o consumo colaborativo e valores como Tradição são menos pontuados pelos 
respondentes. Ao fundamentar-se em uma perspectiva mais recente de Valores Humanos, ela apresenta resultados que poderão suscitar novas discussões a respeito desta temática.

Como anteriormente exposto, os resultados da pesquisa vêm a contribuir de forma inédita sobre o entendimento dos valores humanos no contexto do consumo colaborativo, tendo em consideração a utilização da Teoria Funcionalista vinda da Psicologia Social. Portanto, estudos posteriores que venham a utilizar esse enfoque teórico, poderão se basear nesses achados para propor a construção de hipóteses, como de que pessoas que consomem de forma colaborativa são mais jovens, mais preocupadas com economia de custos, e possuem visão de mundo mais ampla, por exemplo, bem como podem ser formulados modelos diversos que estabeleçam relações entre valores humanos e outras variáveis, dentro do contexto do consumo colaborativo.

Em adição às contribuições teóricas, algumas implicações práticas podem ser tiradas enfatizando pontos pertinentes para os profissionais de marketing. Primeiramente, esses poderiam utilizar a publicidade específica visando capturar valores mais ligados à experimentação de coisas novas, à abertura a mudanças. Por exemplo, o Airbnb já vem fazendo isso ao disponibilizar novos serviços aos seus usuários. Recentemente, foi colocada a opção de realizar atividades nas cidades que os hóspedes irão ficar, como aulas de yoga, culinária, fazer escalada, etc. Essa estratégia é passada para os usuários como novas experiências, isso também concerne ao fato de os consumidores desses serviços procurarem a interação social com os moradores locais. Outro exemplo de algo que poderia ser feito é a respeito da forte motivação de poupança de custos. 0 Airbnb poderia oferecer promoções aos seus usuários mais antigos, descontos na hospedagem, etc.

Para além de tais contribuições, acredita-se que, no atual contexto vivenciado em todo o mundo, em que a pandemia da COVID-19 afeta diretamente setores como saúde pública e economia, ao passo em que isola e distancia as pessoas, isto é, afetando indiretamente também os valores humanos, o consumo colaborativo pode vir a ganhar ainda mais força e notoriedade no comportamento dos consumidores de todo o mundo, por se mostrar não apenas uma solução economicamente viável, o que se mostra bastante necessário e procurado em cenários pós-crise, mas também por proporcionar interações e sociabilidade entre os agentes que estabelecem essa relação de consumo.

Quanto às limitações do estudo, em relação à forma de coleta, é importante a considerar diz respeito à possíveis vieses inerentes ao tipo de medida utilizada (autorrelato). Considerando que construtos como valores podem ser influenciados pela desejabilidade social (Schartz et al., 1997), os participantes podem emitir respostas falsas que acreditem ser mais aceitáveis socialmente. Percebeuse isso quando alguns respondentes vieram questionar a relevância do valor sexualidade para a pesquisa. Possivelmente se sentiram constrangidos de responder com veracidade a importância daquele valor em suas vidas.

Para futuros estudos, sugere-se que utilizando a perspectiva funcionalista, os resultados da pesquisa contendo os valores humanos de consumidores que participam de uma atividade de consumo colaborativo sejam comparados com amostras maiores contendo, por exemplo, os valores humanos da população brasileira.

\section{Referências}

Ajzen, I. (1985). Attitudes, personality and behavior. Chicago: Dorsey Press.

Ajzen, I. (1991). The theory of planned behavior. Organizational Behavior and Human Decisions Processes. University of Massachusetts, Academic Press, 179-211.

Arruda, H. R., Bandeira, E. L., Silva, A., \& Rebouças, A. M. (2016). Consumo colaborativo e valores pessoais: o caso da bicicleta compartilhada. Revista Brasileira de Marketing - ReMark, 15(5).

Athayde, R. A. A. (2012). Medidas implícitas de valores humanos: elaboração e evidências de validade. Dissertação de Mestrado em Psicologia Social. Universidade Federal da Paraíba.

Belk, R. (2007). Why not share rather than own? The ANNALS of the American Academy of Political and Social Science, 611(1), 126-140. 
Belk, R. (2014). You are what you can access: sharing and collaborative consumption online. Journal of Business Research, 67 (8), 1595-1600.

Böcker, L., \& Meelen, T. (2017). Sharing for people, planet or profit? Analysing motivationsfor intended sharing economy participation. Environmental Innovation and Societal Transitions, 23, 28-39.

Bostman, R., \& Rogers, R. (2010). What's mine is yours: the rise of collaborative consumption. Estados Unidos: HarperCollins.

Chen, K. K., Lune, H., \& Queen, E. L. (2013). How values shape and are shaped by nonprofit and voluntary organizations: the current state of the field. Nonprofit and Voluntary Sector Quarterly, 42(58), 856-885.

Cohen, B., \& Kietzmann, J. (2014). Ride on! mobility business models for the sharing economy. Organization \& Environment, 27(3), 279-296.

Cronbach, L. J. (1951). Coefficient alpha and the internal structure of tests. Psychometrika, 16, 297-334.

Da Costa, F. J. (2011). Mensuração e desenvolvimento de escalas: aplicações em administração. Rio de Janeiro: Editora Ciência Moderna.

Gouveia, V. V. (2003). A natureza motivacional dos valores humanos: evidências acerca de uma nova tipologia. Estudos de Psicologia, 8(3), 431-443.

Gouveia, V. V. (1998). La naturaleza de los valores descriptres del individualismo y del colectivismo: una comparación intra e intercultural. Tese de Doutorado. Departamento de Psicologia Social, Universidade Complutense de Madri, Espanha.

Gouveia, V. V. (2013). Teoria funcionalista dos valores humanos: fundamentos, aplicações e perspectivas. São Paulo, Brasil: Casa do Psicólogo.

Gouveia, V. V., Andrade, J. M., Milfont, T. L., Queiroga, F., \& Santos, W. S. (2003). Dimensões normativas do individualismo e coletivismo: é suficiente a dicotomia pessoal vs. social? Psicologia: Reflexão e Crítica, 16, 223-234.

Gouveia, V. V., Milfont, T. L., Fischer, R., \& Santos, W. S. (2008). Teoria funcionalista dos valores humanos. In: Teixeira, M. L. M. (Eds). Valores humanos e gestão: novas perspectivas. São Paulo: Senac, 47-80.

Gouveia, V. V., Milfont, T. L., \& Guerra, V. M. (2014). Functional theory of human values: testing its content and structure hypotheses. Personality and Individual Differences, 60(1), 41-47.

Gouveia V. V., Milfont, T. L., Vione, K. C., \& Santos, W. S. (2015). Guiding actions and expressing needs: on the psychological functions of values. Psykhe, 1-14.

Gouveia, V. V., Fonsêca, P. N., Milfont, T. L., \& Fishcer, R. (2011).Valores humanos: contrinuições e perspectivas teóricas. In: Torres, C. V.; Neiva, E. R. (Eds.). A psicologia social: principais temas e vertentes. Porto Alegre, RS: ArtMed.

Hamari, J., Sjöklint, M., \& Ukkonen, A. (2016). The sharing economy: why people participate in collaborative consumption. Journal of the Association for Information Science and Technology, 67(9), 2047-2059.

Inglehart, R. (1977). The silent revolution: changing values and political styles among western publics. Princeton, NJ: Princeton University Press.

Kathan, W., Matzler, K., \& Veider, V. (2016). The sharing economy: you business model's friend or foe? Business Horizons, 59(6), 663-672.

Kluckhohn, C. (1951). Los valores y las orientaciones de valor en la teoría de la acción. In: Parsons, T.; Shils, E.A. (Orgs.). Hacia una teoría general de la acción. Buenos Aires: Editorial Kapelusz.

Malhotra, N. K. (2006). Pesquisa de marketing: uma orientação aplicada. Porto Alegre: Bookman.

Martin, C., \& Upham, P. (2015). Grassroots social innovation and the mobilisation of values in collaborative consumption: a conceptual model. Journal of Cleaner Production, 134, 204-213.

Maslow, A. (1954). Motivation and personality. New York: Harper and Row.

Milfont, T. L., Gouveia, V. V., \& Da Costa, J. B. (2006). Determinantes psicológicos da intenção de constituir família. Psicologia: Reflexão e Crítica, 19, 25-33.

Möhlmann, M. (2015). Collaborative consumption: determinants of satisfaction and the likelihood of using a sharing economy option again. Journal of Consumer Behaviour, 14, 193-207.

Parsons, T. (1950). El sistema social. Madri: Revista de Occidente. 
Piscicelli, L., Cooper, T., \& Fisher, T. (2015). The role of values in collaborative consumption: insights from a product-service system for lending and borrowing in the UK. Journal of Cleaner Production, 97, 21-29.

Ross, D., \& Hahn, R. (2017). Does shared consumption affect consumers' values, attitudes, and norms? a panel study. Journal of Business Research, 77, 113-123.

Santoso, A., \& Erdaka, A. (2015). Customer loyalty in collaborative consumption model: empirical study of CRM for product-service system-based e-commerce in Indonesia. Procedia Computer Science. 72, $543-551$.

Schwartz, S. H. (1994). Are there universal aspects in the structure and contents of human values? Journal of Social Issues, 50 (4), 19-46.

Schwartz, S. H. (2006). Há aspectos universais na estrutura e conteúdo dos valores humanos? In: Ros, M; Gouveia, V. V. (Eds.). Psicologia social dos valores humanos: desenvolvimentos teóricos, metodológicos e aplicados. São Paulo: Editora Senac, 55-85.

Schwartz, S. H. (1992). Universals in the content and struture of values: theoretical advances and empirical tests in 20 countries. In: Zanna, M. (Ed.). Advances in experimental social psychology, New York, 24, 1-65.

Silva, M. J. B., \& Barbosa, M. L. A. (2018). Compartilhando Bicicletas e Consumindo Experiências: uma investigação do consumo colaborativo praticado por usuários do Bike PE. Consumer Behavior Review, 2(Special Edition), 1-18.

Sousa Júnior, J. H. (2018). "Vai uma carona, aí?": Experiências de consumo colaborativo de usuários de aplicativo de caronas. Consumer Behavior Review, 2(Special Edition), 33-42.

Stern, P. C., Dietz, T., Abel, T., Guagnano, G., \& Kalof, L. (1999). A value-belief-norm theory of support for social movements: the case of environmentalism. Research in Human Ecology, 6(2), 81-97.

Tussyadiah, I. (2015) An exploratory on drivers and deterrents of collaborative consumption in travel. In: Tussyadia, I.; Inversini, A. (Eds.). Information \& Communication Technologies in Tourism. Switzerland, 817-830.

Welzel, C., Inglehart, R., \& Kligemann, H.-D. (2003). The theory of human development: a cross-cultural analysis. European Journal of Political Research, 42, 341-379. 